\title{
Vers un cadre d'analyse interactionniste des pratiques professionnelles
}

\author{
Joëlle Morrissette, Ph.D. ${ }^{1}$
}

\author{
Université de Montréal
}

\begin{abstract}
Résumé
Cette contribution vise à rendre compte d'une avancée méthodologique réalisée dans le cadre d'une recherche doctorale qui a documenté les « manières de faire » l'évaluation formative des apprentissages de cinq enseignantes du primaire. La prise en compte de l'interaction entre les participantes lors des entretiens de groupe, effectuée à partir d'une thématisation du contenu discursif ainsi que d'une analyse de conversations, a en effet permis de dégager un cadre d'analyse susceptible d'éclairer différentes pratiques professionnelles. Ainsi ont été dégagés des conventions d'une culture professionnelle à partir des « manières de faire partagées », des routines et théories-en-usage singulières à partir des «manières de faire admises » et des accords pragmatiques qui montrent comment des praticiennes s' «arrangent» au quotidien avec certaines contraintes institutionnelles ou pressions sociales à partir des «manières de faire contestées ». Cette avancée méthodologique est donc tributaire de la posture interactionniste assumée dans le cadre de cette recherche.

Mots clés

THÉMATISATION DU CONTENU DISCURSIF, ANALYSE DE CONVERSATIONS, CADRE D'ANALYSE INTERACTIONNISTE, PRATIQUE PROFESSIONNELLE, CONVENTIONS, THÉORIES-EN-USAGE, ACCORDS PRAGMATIQUES
\end{abstract}

\section{Introduction}

La tradition de Chicago invite globalement à concevoir le monde social sous l'angle des interactions. Elle a été instaurée à l'Université de Chicago par deux générations de sociologues - autour de 1920-1940 et de 1950-1960 - dont les travaux se sont révélés d'une grande fécondité, comme le soulignent plusieurs auteurs (Chapoulie, 2001; Coulon, 1992; Pessin, 2004; Woods, 1992). En effet, de cette tradition est issue une nouvelle compréhension des problématiques urbaines (par exemple Thomas \& Znaniecki, 1918-1920) et institutionnelles (par exemple Goffman, 1968), ainsi qu'une nouvelle façon d'envisager les mondes du travail (par exemple Hughes, 1951, 1996; Strauss, 1971), de la culture et de l'art (par exemple Becker, 1982/2006b). Connaissant une

RECHERCHES QUALITATIVES - Vol. 30(1), pp. 10-32.

DE L'USAGE DES PERSPECTIVES INTERACTIONNISTES EN RECHERCHE ISSN 1715-8702 - http://www.recherche-qualitative.qc.ca/Revue.html (C) 2011 Association pour la recherche qualitative 
renommée particulière dans les années 1950-1960 sous l'impulsion des recherches de sociologues tels qu'Howard S. Becker, Erving Goffman et Anselm Strauss, cette tradition fait l'objet aujourd'hui d'une redécouverte dans le contexte de ce que d'aucuns appellent le "retour de l'acteur » en recherche (Touraine, 1984). En outre, sa popularité actuelle est manifeste dans la (re)traduction en langue française d'ouvrages des sociologues de Chicago (par exemple Becker, 2006a; Glaser \& Strauss, 1967/2010) ou de l'utilisation de leur héritage pour les projets particuliers d'autres chercheurs francophones, et ce, dans différents domaines.

Comme en ont bien fait état plusieurs (par exemple Becker \& McCall, 1990; Chapoulie, 2001, 2008; De Quieroz \& Ziolkowski, 1997), de cette tradition découle un ensemble hétérogène de perspectives interactionnistes : un interactionnisme symbolique à la Herbert Blumer (1937, 1969), repris et développé par Howard S. Becker, un interactionnisme constructiviste à la Berger et Luckmann (1986) qui, sous l'inflexion des auteurs, devient une théorie de la connaissance, un interactionnisme à tendance réaliste tel que conçu par Goffman (1973), etc. Nonobstant cette diversité de perspectives, les chercheurs qui s'inspirent de cette tradition sociologique ont néanmoins en commun certains intérêts dont l'expérience quotidienne des acteurs, adoptent une vision interprétative du monde et partagent certaines préférences méthodologiques tel le travail de terrain avec des données de première main. Enfin, plusieurs imprègnent leur perspective interactionniste de la sociologie compréhensive de Mead (1863-1931), s’intéressant à ce point de vue «de l'intérieur », et donc à l'univers de significations auquel les acteurs se réfèrent, aux logiques qui sous-tendent leurs actions.

M'inscrivant dans l'héritage intellectuel de cette tradition de recherche, j'ai documenté le savoir-faire de cinq enseignantes du primaire en matière d'évaluation formative des apprentissages des élèves ${ }^{2}$, et ce, à partir de leurs propres points de vue sur leurs pratiques (Morrissette, 2009, 2010a). Si l'ensemble de la recherche a été réalisée à l'aune d'une posture interactionniste inspirée des travaux de Becker $(1982 / 2006 b, 1986)$ - de la phase de problématisation à celle de la présentation des résultats de recherche -, dans le cadre de cette contribution je montrerai comment l'approche analytique interactionniste développée conduit à proposer un cadre d'analyse susceptible d'éclairer différentes pratiques professionnelles ${ }^{3}$. Ainsi, après avoir rapporté quelques éléments se rapportant à la problématique de cette étude qui concerne le rapport qui est établi habituellement à la pratique des enseignantes et des enseignants, j'expliquerai comment, en misant sur les interactions du groupe d'enseignantes lors des entretiens collectifs, j'ai déployé une approche analytique visant à cartographier le territoire de l'évaluation formative par un 
travail de thématisation puis par une analyse de conversations. Comme on le verra avec les exemples proposés, cette approche analytique interactionniste aura ainsi permis de dégager des conventions d'une culture professionnelle à partir des "manières de faire partagées ", des routines ou théories-en-usage singulières à partir des "manières de faire admises » et, enfin, des accords pragmatiques qui montrent comment des praticiennes s' «arrangent» au quotidien avec certaines contraintes institutionnelles ou pressions sociales à partir des «manières de faire contestées ».

\section{La problématique de l'étude : faire de la recherche « avec » et non «sur "}

L’étude doctorale réalisée (Morrissette, 2009, 2010a) s'inscrit dans la foulée des travaux d'auteurs tels que de Certeau (1990) ou Giddens (1987) qui soutiennent que les gens « ordinaires » produisent, eux aussi, des savoirs et des «manières de faire » avec et dans la culture. Elle s’inscrit également dans la foulée du mouvement de démocratisation de l'expertise auquel participent Callon, Lascoumes et Barthe (2001), Van Campenhoudt, Chaumont et Franssen (2005), Lieberman (1986) ou encore Darré (1999) qui reconnaissent que les acteurs peuvent contribuer à la production de savoirs à propos de problèmes et d'enjeux qui les impliquent. En cela, les options retenues s'éloignent des manières de dire et de faire habituelles dans le domaine de l'évaluation des apprentissages où est généralement véhiculée une vision instrumentale et techniciste de la mise en œuvre de l'évaluation formative et où les pratiques sont souventes fois jugées à l'aune de modèles théoriques développés dans les milieux universitaires « aseptisés », décontextualisés.

Renversant cette perspective prescriptive et normative qui relève du modèle de la rationalité technique dénoncé par Schön (1996), je me suis intéressée au savoir-faire d'enseignant(e)s du primaire en matière d'évaluation formative des apprentissages à partir de leurs propres points de vue. Pour ce faire, je me suis inspirée du modèle d'approche collaborative imaginé par Desgagné (1997, 2001; Morrissette \& Desgagné, 2009) qui vise à proposer à des praticiens un processus d'accompagnement au développement professionnel (formation continue) dans le cadre d'une démarche formelle de recherche. Ce modèle repose sur l'idée selon laquelle leurs savoirs pratiques peuvent être mis sous une forme discursive dans le cadre d'un dispositif de collaboration entre chercheurs et praticiens qui prend la forme d'activités réflexives où se coconstruit un objet de recherche à partir d'une réflexion partagée sur l'expérience pratique. Ainsi, dans une telle approche, le chercheur ne se positionne pas en expert de l'objet, comme dans des formations plus traditionnelles, mais en facilitateur de l'explicitation de savoirs pratiques qui 
sont en grande partie tacites (Schön, 1983). Autrement dit, une approche collaborative vise la coconstruction d'un savoir professionnel, produit combiné et inédit des logiques, intérêts et enjeux des uns et des autres. En ce sens, ce modèle peut être vu comme visant d'une certaine manière à concilier le monde de la pratique des chercheurs universitaires avec celui de la pratique de terrain, visant par là même une "interfécondation des deux cultures » (Desgagné, 1998).

Cinq enseignantes du primaire (de la $4^{\mathrm{e}}$ à la $6^{\mathrm{e}}$ année) ont participé à cette recherche qui privilégiait un rapport non prescriptif, le contrat collaboratif passé avec elles les invitant à s’engager dans une relation de complémentarité. Il s'agit d'enseignantes « ordinaires » qui se sont portées volontaires à la suite de la présentation du projet à l'ensemble des enseignants de l'école. Ainsi, aucune sélection n'a été opérée relativement à leur niveau d'enseignement, à leur nombre d'années d'expérience ou encore à leur réputation (reputational method of selection, Hunter, 1953). Concrètement, trois types d'activités réflexives ont été proposées en alternance (de juin à décembre 2006). J'ai produit des bandes vidéo dans leur classe (3) en leur demandant de les visionner pour elles-mêmes et, le cas échéant, d'y identifier des épisodes d'évaluation formative. Puis des entretiens individuels (3) ont eu lieu, reposant sur un protocole de rétroaction vidéo appelé "réflexion partagée » (Tochon, 1996), susceptible de favoriser la coconstruction de savoirs utiles au développement professionnel. Lors de ces entretiens, les participantes étaient invitées à présenter les épisodes identifiés et à expliciter leurs "manières de faire » (de Certeau, 1990), c'est-à-dire à rendre explicite le rationnel de leur expérience. Ces deux types d'activité faisaient partie d'un contexte préparatoire aux entretiens de groupe (5) réalisés en alternance, dans le cadre desquels les enseignantes étaient invitées à rapporter des épisodes d'évaluation formative ainsi qu'à commenter les pratiques narrées par leurs pairs, à en négocier le sens, voire à en débattre, et ce, à partir de l'ancrage dans l'expérience favorisé par les entretiens individuels.

\section{Les entretiens de groupe : une réflexivité qui s’approfondit dans l'intersubjectivité}

Selon plusieurs, dont Poupart (1997) et Demazière (2008), les entretiens sont conçus comme l'un des meilleurs moyens pour coconstruire avec les acteurs le sens qu'ils donnent à leurs conduites et pour étudier la façon dont ils se représentent le monde, ce qui suppose de reconnaître qu'ils sont les mieux placés pour en parler. De manière plus particulière, l'entretien de groupe constitue un choix pertinent en vue d'investiguer le sens commun, les modèles culturels et les normes partagés, de même que des dimensions plus 
conflictuelles, par la prise en compte des interactions qui se manifestent dans les discussions des groupes recrutés (Duchesne \& Haegel, 2005).

Le choix de centrer la démarche d'investigation de manière particulière sur les entretiens de groupe repose principalement sur le postulat de la fécondité des interactions qu'ils engagent entre les participants (Morrissette, 2011). De fait, les négociations sociodiscursives permettent l'émergence de dimensions imprévues relatives à l'objet d'étude - ce que n'autorise pas le modèle de la rationalité technique -, davantage que ne le font les entretiens individuels qui encouragent une dynamique «questions-réponses » susceptible d'entraîner la production d'énoncés somme toute prévisibles. En outre, en ayant pour objectif de mieux comprendre une pratique professionnelle, il a semblé que le choix le plus fondé soit celui de proposer à certains de ses membres de discuter autour de cette pratique, d'en négocier le sens.

Les entretiens de groupe, comme ceux conduits dans le cadre de cette investigation, peuvent être conçus telles des conversations, soit des événements interactionnels en tension entre un cadre conventionnel constitué de systèmes d'attentes mutuelles qui amènent une certaine prévisibilité des échanges, et la négociation du sens et des règles conversationnelles en continu qui sont susceptibles de laisser émerger des aspects non anticipés (Goffman, 1987; Gumperz, 1989; Kerbrat-Orrecchioni, 1996). Également, les entretiens de groupe donnent à voir le langage commun véhiculé entre membres d'un groupe partageant les mêmes activités, langage qui, référant aux propositions de Giddens (1987) ou Wittgenstein (2004, 2006), serait susceptible de rendre compte de la façon dont est conçue la pratique. En ce sens, les entretiens de groupe seraient porteurs des savoirs utiles aux activités quotidiennes organisées des membres. Enfin, choisir l'entretien de groupe, c'est aussi faire le pari de la fécondité du métissage des points de vue pour explorer l'objet d'étude, les interactions permettant à des praticiens de soumettre le sens dégagé de leur expérience à la médiation de leurs pairs, le croisement des regards favorisant l'exploration de la complexité de la pratique.

\section{Le projet analytique : cartographier le territoire d'une pratique}

En prenant appui sur la métaphore de la carte routière proposée par Fourez (2003), on peut considérer que le projet analytique de cette recherche en était un de cartographie du territoire de l'évaluation formative : la carte n'est pas le territoire, mais en tient lieu, elle en constitue une représentation, et permet de cheminer dans l'univers symbolique construit dans les processus d'explicitation négociée des manières de faire du groupe d'enseignantes. En ce sens, c'est une posture dite "analytique " (Demazière \& Dubar, 1997) ${ }^{4}$ qui préside à ce projet, posture selon laquelle ce sont les influences théoriques qui 
donnent forme au sens qui est dégagé des entretiens. Cette posture se révèle pertinente dans le cadre de cette recherche spécifique qui vise à conceptualiser les façons de mettre en œuvre l'évaluation formative des enseignantes par une approche compréhensive de leurs points de vue. Comme on le verra dans la suite, ce projet de cartographie a été concrétisé par le déploiement d'une approche analytique constituée de deux mouvements visant à surmonter un problème méthodologique qui s’est posé.

\section{L'approche analytique initiale : intégrer la logique des acteurs de terrain}

L'approche analytique initiale a consisté en une thématisation du contenu discursif, soit un processus de catégorisation ouvert, sans catégories a priori, guidé par cette question: De quoi parlent les enseignantes lorsqu'elles conversent autour de leurs pratiques d'évaluation formative? Cette première étape a permis de dégager des dimensions (12) auxquelles les enseignantes lient leurs pratiques d'évaluation formative, et donc de faire une première cartographie du territoire. À l'instar de ce que propose Van der Maren (2004), ces dimensions ont été regroupées sous deux grandes catégories qui respectent celles qui étaient toujours présentes dans les épisodes narrés par les praticiennes. La première catégorie, nommée «manières de définir la situation des élèves face aux apprentissages » en référence au concept de «définition de la situation " (Thomas, 1923), regroupait un ensemble de pratiques générales (6) par lesquelles les enseignantes disaient procéder à l'élaboration d'un jugement visant à identifier des obstacles d'apprentissage ou encore à reconnaître le savoir-faire des élèves à l'égard de certains objets d'apprentissage. Par exemple, apprécier les démarches employées par les élèves dans toutes leurs productions quotidiennes ou encore décoder leur nonverbal semblaient constituer des façons privilégiées de "définir la situation ». La seconde catégorie, nommée "manières de soutenir les apprentissages ", regroupait un ensemble de pratiques générales (6) par lesquelles les enseignantes disaient soutenir les apprentissages des élèves à partir de leur évaluation de la situation, l'aspect «intervention » constituant pour elles une condition sine qua non de l'évaluation formative. Par exemple, susciter la réflexion chez les élèves ou encore réaménager les contextes d'enseignement/apprentissage ont été identifiés comme des «manières de soutenir les apprentissages » (voir Figure 1).

Pour chacune de ces 12 pratiques générales, un ensemble de pratiques spécifiques a été identifié. À titre illustratif, susciter la réflexion chez les élèves en tant que «manière de soutenir les apprentissages » renvoie à six (6) pratiques spécifiques différentes (voir Tableau 1). 


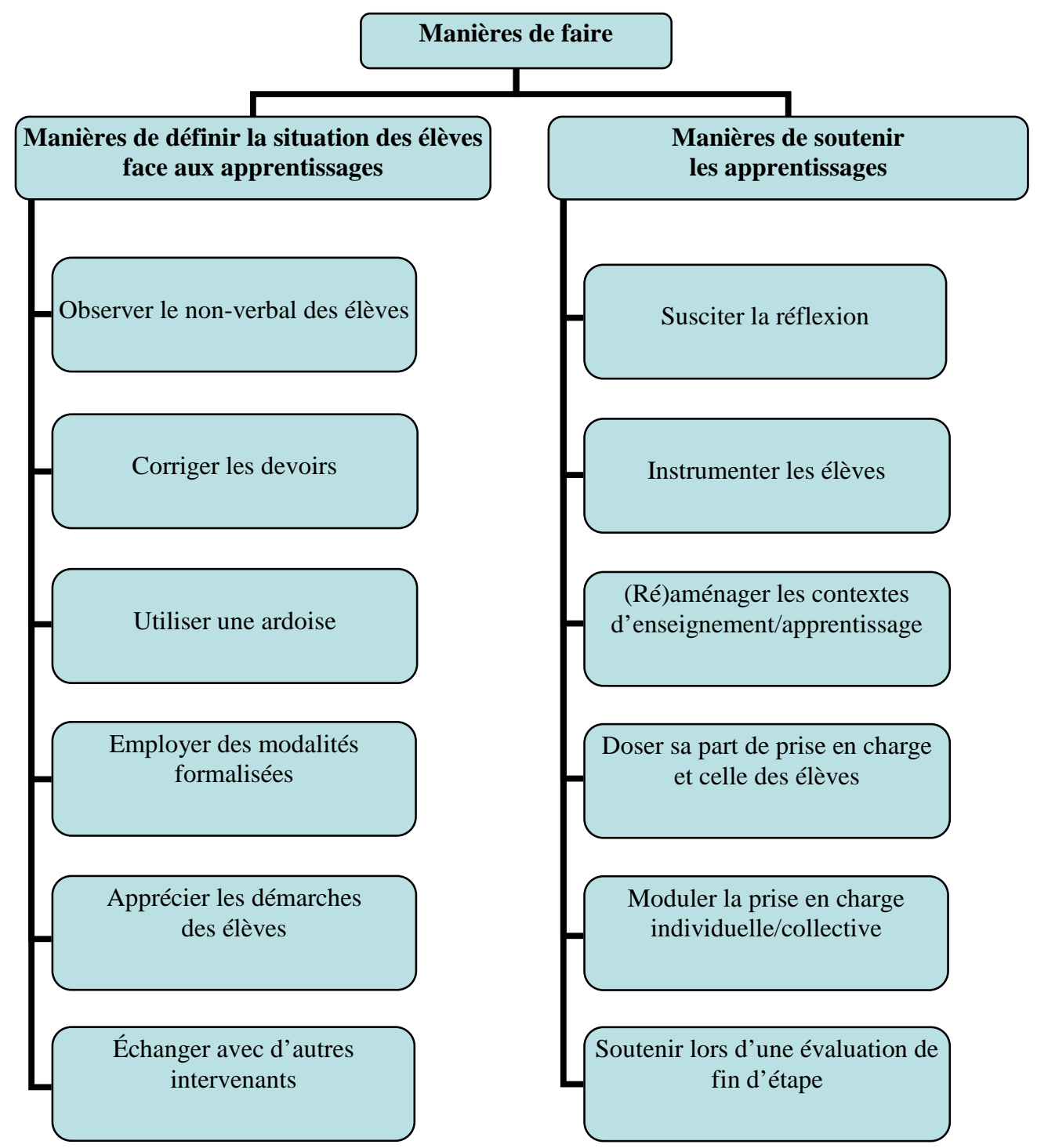

Figure 1. Manières de faire l'évaluation formative 
Tableau 1

Susciter la réflexion chez les élèves

Questionner en continu pour stimuler et guider la réflexion des élèves.

Laisser le raisonnement des élèves s'engager dans des impasses par rapport à un problème donné.

Déstabiliser les élèves pour ébranler leurs façons de faire habituelles et susciter leur engagement.

Utiliser le portfolio pour amener les élèves à évaluer leurs progressions.

Inscrire la rétroaction dans une logique d' «après-coup».

Employer un code de rétroaction avec des lettres.

\section{Un problème méthodologique : l'effet " liste d'épicerie "}

Nonobstant l'intérêt qu'aurait pu revêtir une présentation des résultats selon cette logique énumérative (cf. Figure 1), qui constitue déjà une contribution au domaine de l'évaluation formative, la thématisation du contenu discursif s'est révélée être une stratégie insuffisante car elle donnait à penser que les enseignantes n'avaient qu'établi une liste de leurs manières de faire. Il n'en est rien! Elles se sont engagées dans des processus de négociation autour des pratiques explicitées, et il fallait donc définir une seconde stratégie analytique pour en témoigner. Ainsi, pour rendre justice à la façon dont l'objet a été coconstruit en situation d'entretien de groupe, m'appuyant sur le point de vue interactionniste adopté selon lequel le sens construit est indissociable de la façon dont il est produit dans l'interaction entre les enseignantes, j'ai examiné le contexte d'énonciation autour de l'explicitation de chacune des manières de faire (Morrissette, 2010b). À cette fin, une analyse de conversations inspirée en partie des travaux de Boden (1990) a été réalisée afin d'étudier minutieusement comment s'est coconstruit le savoir sur l'évaluation formative. L'attention, lors de ce deuxième mouvement analytique, s'est centrée sur la "négociation conversationnelle »- un concept emprunté à Kerbrat -Orrecchioni (2005) -, soit les processus de coopération entre les enseignantes autour de l'explicitation de leurs manières de faire. Dans le cadre de l'exemple qui suit, tiré de l'un des verbatims des entretiens de groupe, on voit bien que l'interaction est composée de processus de reconnaissance mutuelle qui permettent d'interpréter que les enseignantes partagent l'expérience de la pratique spécifique en cause, soit 
laisser le raisonnement des élèves s'engager dans une impasse comme façon d'intervenir dans une perspective formative :

P3 : [...] je déstabilise les enfants; par exemple, hier, un élève a fait un problème de mathématiques, et puis sa démarche était erronée; je ne disais pas un mot, je le laissais aller, et je regardais la réaction des autres élèves

P4 : souvent, les autres élèves ne réagissent pas!

P3 : aucun d'entre eux n'a réagi! à un moment donné, un élève a fini par dire : «il me semble que ce n'est pas comme ça qu'il faut faire »; j’ai dit : « ah non?! »

P1 : on les laisse mener un raisonnement erroné jusqu'au bout

P3 : oui, et on voit les autres élèves qui ne disent pas un mot!

P2 : en fait, ils te regardent en espérant que tu dises : «ça ne marche pas! »

P3 : oui, oui!

P1 : certains élèves ne sont pas sûrs

P3 : et là tu les laisses s'embourber, et quelques-uns sont déstabilisés et se questionnent sur leur démarche parce qu'elle est différente de celle présentée

P5 : ils ne comprennent pas pourquoi on ne réagit pas, pourquoi on les laisse s'engager dans une impasse

P4 : c'est ça! moi aussi je fais ce type d'intervention

Ainsi, cette attention portée à la façon dont se sont négociées les manières de faire lors des entretiens de groupe a ainsi conduit à distinguer :

- des «manières de faire partagées », soit celles qui ont suscité des processus de reconnaissance mutuelle dans l'interaction, des constructions consensuelles;

- des «manières de faire admises ", soit celles qui ont fait l'objet d'une explicitation par une enseignante, sans marque d'adhésion ou d'objection de la part de ses pairs;

- des «manières de faire contestées », soit celles qui ont soulevé des désaccords persistants entre les enseignantes, n’étant pas reconnues d'emblée comme une pratique d'évaluation formative.

Précisons au sujet des «manières de faire contestées" que les désaccords persistants entre les enseignantes ont été distingués de simples ajustements de sens dans l'interaction, à la suite des propositions de KerbratOrecchioni (2005) et de Traverso (1999) : lorsqu'une proposition est émise par une première enseignante et que survient une contestation de cette proposition par une deuxième enseignante, laquelle est souvent assortie d'une contre- 
proposition, il y a un simple "ajustement » dans l'interaction si la première enseignante accepte immédiatement cette contre-proposition ou si elle décide de passer outre plutôt que de focaliser sur le différend; au contraire, un désaccord s'amorce lorsque la première enseignante rejette cette contre-proposition et maintient sa position initiale, ce qui, en quelque sorte, le cristallise, comme le font remarquer les auteures.

\section{L'approche analytique interactionniste: la description d'un territoire de pratique}

La combinaison de ces deux mouvements analytiques a ainsi permis, d'une part, de décrire de façon nuancée le territoire de l'évaluation formative des apprentissages selon le groupe d'enseignantes rencontrées. Reprenant l'exemple précédent, susciter la réflexion chez les élèves en tant que «manière de soutenir les apprentissages " s'est scindé, en fonction de l'interaction du groupe de pairs, en six pratiques spécifiques dont certaines sont partagées, l'une admise et d'autres contestées ${ }^{5}$ (voir Tableau 2).

D'autre part, en dégageant trois « zones territoriales », la combinaison de ces deux mouvements analytiques a également permis de dégager des conventions de la culture professionnelle des enseignantes à partir des «manières de faire partagées », des singularités telles des routines ou des théories-en-usage à partir des "manières de faire admises » et, enfin, des accords pragmatiques permettant de négocier des tensions entre la visée de soutien aux apprentissages de l'évaluation formative, les contraintes institutionnelles et les pressions sociales à partir des «manières de faire contestées ».

\section{La zone partagée : des conventions d'une culture professionnelle}

À partir des travaux de Becker (1982/2006b), la zone partagée peut être associée aux pratiques qui relèvent des conventions de la culture des enseignantes, c'est-à-dire des savoirs pratiques de leur groupe professionnel qui leur permettent de se livrer à leurs activités quotidiennes de soutien aux apprentissages de leurs élèves par le biais d'une évaluation formative. Identifier ces conventions permet de saisir les allant-de-soi de la culture enseignante, c'est-à-dire ce qui n'est pas remis en question, tel un "fonds de commerce » sur lequel les enseignantes, du moins celles rencontrées, s’appuient au quotidien pour soutenir les apprentissages de leurs élèves par l'entremise de processus évaluatifs.

Parmi les conventions identifiées, j’ai notamment trouvé certaines « réifications » (re)produites par le groupe d'enseignantes, soit des concepts qui sont " chosifiés », au sens convenu depuis Bachelard, et donc détachés de leurs 
Tableau 2

Susciter la réflexion chez les élèves : manières de faire partagées, admises et contestées

Questionner en continu pour stimuler et guider la réflexion des partagée élèves.

Laisser le raisonnement des élèves s’engager dans des impasses par rapport à un problème donné.

partagée

Déstabiliser les élèves pour ébranler leurs façons de faire

admise habituelles et susciter leur engagement.

Utiliser le portfolio pour amener les élèves à évaluer leurs progressions.

contestée

Inscrire la rétroaction dans une logique d' «après-coup».

contestée

Employer un code de rétroaction avec des lettres.

contestée

conditions sociales de production. Par exemple, les catégories d'élèves constituent des réifications: lors de l'explicitation des différents épisodes d'évaluation formative, les enseignantes parlent des «élèves forts et des faibles » - ou des «élèves en difficulté ». Comme invite à l'envisager Becker (1982/2006b), cette convention semble faciliter le travail quotidien, constituant un élément important en vue de la planification de leur enseignement et de l'organisation des interactions visant à favoriser les apprentissages. En effet, lorsqu'elles explicitent certaines pratiques spécifiques d'évaluation formative, par exemple celle qui consiste à aménager des espaces de dialogue entre élèves, les enseignantes affirment le faire souvent pour amener "les faibles ", ceux qui sont à risque d'échec scolaire, à s'exprimer pour voir s'ils ont des difficultés, ou pour permettre « aux forts » de modéliser des démarches autres, peut-être plus viables, avec «des mots pour le dire d'une autre façon » (eg3, 1294-1295). En somme, elles miseraient sur la mise en place et l'exploitation de la dynamique entre ces deux catégories d'élèves comme façon d'intervenir dans une perspective formative.

Un autre exemple de convention identifiée a trait à la mobilisation de schèmes interprétatifs culturels issus, selon différents auteurs (Giddens, 1987; Wenger, 2005), de la graduelle intégration de concepts, de théories et de procédures qui ont fait leurs preuves au sein d'une culture professionnelle historiquement et socialement située. Ainsi, un des schèmes dégagés de la zone 
partagée réside dans la vision plutôt socioconstructiviste des processus d'enseignement/apprentissage qui domine chez les enseignantes. Comme l'affirme Legendre (2001), une pédagogie socioconstructiviste accorde notamment un rôle central aux élèves dans leurs processus d'apprentissage et reconnaît l'importance des interactions en classe dans une démarche de coconstruction des connaissances. Reprenant l'exemple cité précédemment, lorsqu'elles aménagent des espaces de dialogue entre élèves comme pratique spécifique d'évaluation formative, les enseignantes misent sur la dynamique des échanges, voire sur la confrontation des points de vue, pour constituer des obstacles à négocier ou pour amener les élèves à envisager des perspectives différentes des leurs. Dans ce contexte, elles se positionnent en médiatrices des échanges pour laisser le leadership de la réflexion à conduire sur les activités à différents élèves qui, tour à tour, se succèdent à l'avant-scène; parfois aussi, elles se positionnent en animatrices d'une discussion à laquelle tous contribuent. Les enseignantes conçoivent que laisser des élèves argumenter et débattre, s'expliquer dans leurs propres mots, est susceptible de favoriser le soutien aux apprentissages, reconnaissant au groupe-classe une certaine capacité régulatrice. Enfin, on voit bien le mode de représentation plutôt socioconstructiviste mobilisé lorsqu'elles disent «laisser le raisonnement des élèves s'engager dans des impasses par rapport à un problème donné ", leur laissant la responsabilité collective de négocier leurs différents points de vue sur la façon de s'en sortir.

\section{La zone admise : des pratiques singulières et créatrices}

La zone admise regroupe des manières de faire qui, sans être nécessairement partagées par l'ensemble des enseignantes, sont reconnues comme faisant partie du territoire de l'évaluation formative. Toujours en prenant appui sur les travaux de Becker (1963/1985, 1982/2006b), ces pratiques admises sont envisagées comme des pratiques singulières créatives développées à partir d'expériences personnelles qui se sont révélées satisfaisantes au fil des années. Les "manières de faire admises " se sont manifestées en termes de routines d'intervention formative centrées sur la différenciation pédagogique ainsi qu'en termes de théories-en-usage (Giddens, 1987) ou, dit plus simplement, de principes pédagogiques liés à l'évaluation formative.

Parmi les routines identifiées, prenons celle de l'une des enseignantes qui affirme l'avoir mise en place en raison du nombre élevé d'élèves par classe (29 à 31 élèves de 11 et 12 ans). En effet, le mode d'apprentissage en groupe encourage le plus souvent des moments d'enseignement collectif qui ne permettent qu'une évaluation approximative des progressions des élèves, telle une «prise de pouls » de l'ensemble du groupe. Pour pallier cette situation, 
cette enseignante aménage un temps de prise en charge individuelle à la dernière heure de chaque journée qui est consacrée à la réalisation et à la correction des devoirs en classe - et non à la maison -, soit l'option retenue par ses pairs. Durant cette heure, les élèves travaillent généralement seuls; ils interpellent l'enseignante, au besoin, par un système de gestion des sollicitations ou sont directement convoqués à son bureau. Pour l'enseignante, qui reçoit tour à tour chaque élève, il s'agit d'un moment privilégié pour se pencher sur les réalisations individuelles et surtout pour discuter avec chacun afin de cerner précisément ce qui pose problème, le cas échéant. Concrètement, ce "tête-à-tête » requiert que l'enseignante guide l'élève afin qu'il explicite d'abord ce qu'il comprend, puis ce qu'il ne comprend pas, cette étape étant facilitée par sa façon de tester auprès de lui des hypothèses visant à cerner le problème à partir de leurs avancées respectives. Ainsi, cette routine quotidienne d'évaluation formative suggère que la définition du problème auquel fait face l'élève et le soutien visant à l'aider à le surmonter relèvent d'un processus de coconstruction de sens dans l'interaction.

Quant aux théories-en-usage dégagées des «manières de faire admises », dans une perspective interactionniste, elles sont considérées comme des façons typiques de définir les situations qui engagent certaines conduites régulières de la part des acteurs en fonction des projets particuliers qui les animent. Celles qui ont été dégagées renvoient à des principes pédagogiques liés à la mise en place des conditions jugées essentielles aux apprentissages. Par exemple, l'une des enseignantes a réitéré que pour qu'il y ait apprentissage, l'une des interventions formatives particulières à privilégier consiste à déstabiliser les élèves pour ébranler leurs façons de faire habituelles et susciter leur engagement. Selon elle, il est important de créer une sorte de rupture susceptible de bousculer les conceptions des élèves, afin de "casser » ce qu'elle conçoit comme une habitude de passivité chez eux. Ce principe pédagogique, qui viserait notamment à rendre les apprentissages significatifs pour les élèves, s'actualise, entre autres, en les laissant s'engager dans une activité pour laquelle ils n’ont pas les acquis nécessaires. Par exemple, elle leur propose un jeu avec la calculatrice qui requiert la maîtrise des caractères de divisibilité; leurs essais infructueux feraient émerger chez eux l'intérêt d'apprendre ces caractères afin de pouvoir jouer de façon stratégique. En outre, ce principe pédagogique viserait également à développer chez les élèves une attitude plus réflexive par rapport à leurs démarches d'appropriation des objets d'apprentissage ciblés. Pour ce faire, cette enseignante utilise principalement des techniques dites d'impact (Beaulieu, 2006). L'un des épisodes d'évaluation formative qui en témoigne traite d'un retour sur une tâche mathématique réalisée par les élèves dans le cadre de laquelle ils devaient être capables de 
classer divers polygones. L'analyse des réalisations des élèves amène l'enseignante à interpréter qu'ils ont accompli la tâche sans la comprendre et, surtout, sans l'interpeler dans le but d'avoir des explications supplémentaires. Devant ce constat, elle déploie une technique d'impact comme pratique d'intervention formative : en leur demandant d'aller à la recherche d'un objet inexistant, qu'elle nomme sur-le-champ un « bouli » ${ }^{6}$, elle les amène à prendre conscience qu'il est préférable de se questionner avant même de se lancer dans l'action, et de signaler ses incompréhensions, le cas échéant.

\section{La zone contestée : des accords pragmatiques}

La zone contestée est celle qui donne à voir les pratiques qui interrogent les frontières du territoire de l'évaluation formative. Comme on le verra, la confrontation des différents positionnements qui caractérisent cette zone provoque l'émergence, dans les conversations, de tensions auxquelles se greffent des enjeux. En effet, les contestations des unes et des autres mettent toujours en tension certaines considérations qui lient la finalité de soutien aux apprentissages de l'évaluation formative à des contraintes institutionnelles fortes, à des pressions exercées par certains consensus sociaux. Dans le contexte de cette mise en tension, lorsque les enseignantes objectent un point de vue relativement à une pratique spécifique, elles mobilisent des enjeux comme ressources discursives qui semblent impliqués dans des façons de composer avec cette tension, des façons qui prennent la forme d'activités standardisées, "durcies », a priori non négociables. En élevant l'angle d'analyse au-dessus des différentes prises de position, il se dégage de leurs conversations des «accords pragmatiques» qu'elles bricolent pour s'accommoder des normes liées au réseau d'activités auquel elles participent. L'expression est de Pépin (1994) et renvoie à l'idée d'une interaction efficace sans la nécessité d'un consensus ni même d'une compréhension commune : «il suffit que l'interaction permette à chaque partenaire de construire (déconstruire et reconstruire) le comportement de l'autre de façon viable pour lui-même » (p. 68). Ainsi, en cohérence avec la réflexion posée jusqu’à présent qui montre bien que les enseignantes développent un "savoir y faire » viable même dans un contexte qui peut être défini comme problématique, l'analyse de la zone contestée met aussi en relief qu'elles s' «arrangent », car elle fait ressortir les façons par lesquelles elles s'accommodent d'un tel contexte en maintenant le cap sur la visée de l'évaluation formative.

Par exemple, l'un des accords pragmatiques dégagés consiste à développer des langages communs au service de la différenciation dans le contexte où les enseignantes assument la responsabilité de groupes d'élèves hétérogènes. Lessard et Tardif (1999) traitent d'ailleurs du phénomène de 
diversification de la population scolaire occasionné par la démocratisation de l'école dans les années 1960 au Québec, et exposent de façon particulière l'intégration dans les classes "régulières » d'élèves de diverses cultures et d'élèves ayant des problématiques particulières. Cette situation mettrait en tension des objets d'apprentissage et des critères d'évaluation normatifs avec la question de la différenciation qui est au cœur de l'évaluation formative. En effet, d'une part le curriculum commun exerce une pression vers une uniformisation et, d'autre part, les enseignantes sont en présence d'élèves singuliers qui ont parfois besoin de temps supplémentaire, d'explications différenciées, de matériels pédagogiques distincts, etc. En ce sens, l'enjeu au cœur de cette tension est relatif à la progression de chaque élève dans le groupe. En effet, en ayant à amener tout le groupe d'élèves à réaliser les mêmes apprentissages pour un niveau d'enseignement donné, les enseignantes se heurtent à l'impossibilité d'y parvenir pour certains d'entre eux lorsqu'ils sont placés dans des conditions similaires, voire dans des conditions aménagées spécifiquement pour eux. Elles soutiennent d'ailleurs ne pas toujours parvenir à créer les conditions propices à leurs apprentissages, les limites de leurs propres conditions de pratique rencontrant les limites des élèves dans le contexte scolaire actuel.

L'accord pragmatique déployé par les enseignantes pour négocier la tension qui lie les critères d'évaluation normatifs au soutien différencié des apprentissages VISÉ par l'évaluation formative consiste à définir et à employer des langages communs partagés dans la classe tels que l'ardoise. Lors d'une leçon de groupe, l'ardoise - un carton plastifié sur lequel les élèves peuvent répondre aux questions posées - permet aux enseignantes de "prendre fréquemment le pouls de leur groupe d'élèves » (eg2, 1426-1435), de suivre l'effet approximatif de leur enseignement au fur et à mesure que progresse la leçon, et ce, afin de définir les interventions subséquentes. En outre, l'utilisation de l'ardoise leur permettrait aussi d'identifier les élèves qui ont besoin d'une aide supplémentaire au terme du travail réalisé avec l'ensemble de la classe. À ce titre, l'emploi de l'ardoise constituerait une modalité de rétroaction mutuelle partagée, rapide, au cœur d'une évaluation formative collective susceptible de conduire à des interventions personnalisées dans le contexte d'une leçon de groupe qui ne favorise pas d'emblée la différenciation. Ainsi, les enseignantes miseraient sur le développement du partage de sens autour de cette forme de langage commun pour favoriser l'ajustement mutuel dans une perspective formative, un langage qui induit certaines structures de participation adaptées au contexte du mode d'apprentissage en groupe qui caractérise la forme scolaire actuelle. 


\section{Une objectivation du cadre d'analyse}

Prenant un peu de recul sur les potentialités du cadre d'analyse proposé, il convient de préciser que la zone admise comporte une part d'indéterminé. En effet, pour des raisons qui leur appartiennent, certaines enseignantes ont pu choisir de ne pas dire qu'elles partageaient une pratique spécifique explicitée par l'une de leurs pairs ou qu'elles ne considéraient pas cette pratique comme relevant de l'évaluation formative; un chercheur ne contrôle pas la façon dont les membres d'un groupe de pairs choisissent de se "mettre en scène » (Goffman, 1973). Par ailleurs, des recoupements méthodologiques peuvent diminuer cette part d'indéterminé. De fait, certaines manières de faire qui semblaient de prime abord admises lorsque narrées lors d'un événement conversationnel particulier, ne faisant pas l'objet de marques de reconnaissance mutuelle, étaient finalement considérées comme étant partagées lorsque des liens étaient faits entre différents entretiens collectifs et individuels.

Enfin, si ce cadre d'analyse se veut un moyen de tracer une cartographie du territoire du point de vue des acteurs concernés, il ne saurait prétendre à stabiliser ce qui est en soi dynamique. De fait, la pratique, celle des enseignants ou d'autres groupes professionnels, est en constante évolution et ne saurait être prisonnière d'un portrait figé. De plus, il faut considérer que les entretiens de groupe constituent des événements interactionnels dans le cadre desquels les participants s'interinfluencent, comme l'ont relevé plusieurs (Blanchet \& Gotman, 1992; Mishler, 1986).

\section{Conclusion}

L'approche analytique interactionniste développée dans le cadre de cette recherche doctorale, centrée sur les façons par lesquelles un groupe de pairs collaborent à la production d'un savoir professionnel, a permis la description nuancée d'un territoire de pratique, distinguant des manières de faire partagées, admises et contestées. Ce faisant, elle a mis en relief un éventail de savoir-faire collectifs et singuliers en matière d'évaluation formative des apprentissages. En outre, cette approche a permis de montrer que les savoir-faire d'un groupe sont à la fois tributaires d'une culture professionnelle (zone partagée), d'une capacité à innover, à exploiter une marge de manœuvre personnelle (zone admise) et, enfin, d'une flexibilité sur le terrain qui permet de transformer des contraintes en ressources (zone contestée).

Cela dit, on peut envisager que ce cadre d'analyse interactionniste serait susceptible d'apporter un éclairage sur d'autres types de pratiques professionnelles. Non seulement conduit-il à cerner les pratiques conventionnelles, celles du quotidien, mais il accorde également une place aux pratiques innovantes, celles mises en œuvre par les francs-tireurs dirait Becker 
(1982/2006b). Enfin, il autorise la prise en compte des "cas négatifs », des « cas déviants ", suivant un principe analytique d'importance pour quiconque associe ses travaux à la tradition sociologique de Chicago (Becker, 2002; Woods, 1992). De fait, la «zone contestée " semble composée d'exceptions aux règles d'un groupe dans leur interaction avec des pratiques normalisées et normalisatrices, et donc des manières de faire qui ébranlent les lieux communs ou idées reçues pour ses membres. Ainsi, au lieu d'écarter les cas jugés « hors norme ", ce cadre d'analyse incite plutôt à tenter de comprendre comment et par quoi ils sont reliés à l'objet, favorisant l'exploration de la complexité d'une pratique professionnelle et des enjeux qu'elle recouvre.

\section{Notes}

${ }^{1}$ L'auteure remercie le Conseil de recherche en sciences humaines du Canada (CRSH) dont le financement, sous la forme d'une bourse d'excellence, a rendu possible la réalisation de la thèse à partir de laquelle est tiré cet article.

${ }^{2}$ Globalement, l'évaluation formative, qu'elle soit formalisée dans le cadre d'une tâche papier ou qu'elle revête des formes plus informelles telles que le questionnement continu dans l'interaction de la classe, renvoie à cette fonction de l'évaluation centrée sur les processus d'apprentissage des élèves, susceptible de les soutenir plutôt que de les sanctionner (Morrissette, 2002, 2009).

${ }^{3}$ L'idée de pratiques professionnelles n'est pas à distinguer de ce qu'on appelle par convention des métiers; ici, l'emploi du mot professionnelles renvoie simplement à l'idée du travail d'un groupe de personnes. Ainsi, le cadre d'analyse avancé dans le cadre de cette contribution pourrait servir tout autant à éclairer les pratiques de médecins que de menuisiers.

${ }^{4}$ Demazière et Dubar (1997) distinguent la posture analytique des postures restitutive et illustrative. La posture dite restitutive accorderait une place centrale à rendre compte de la parole des acteurs «telle qu'ils la livrent», c'est-à-dire qu'elle n'est pas transformée à des fins d'exemplification conceptuelle, mais bien considérée comme l'expression transparente des pratiques. Dans cette perspective, l'analyste conçoit que la parole parle d'elle-même, qu'elle est une expression transparente des pratiques des auteurs de cette parole. Quant à la posture dite illustrative, elle reposerait sur la prémisse selon laquelle la parole des acteurs est aveugle sur ce qu'elle a à dire; c'est la théorie qui serait susceptible d'éclairer l'objet de recherche. Sur cette prémisse, l'analyste opère un usage sélectif des paroles des acteurs sollicités en fonction des idées qu'il veut mettre à l'avant-plan en découpant le matériau d'analyse selon des catégories préétablies. Dans cette optique, son projet global est plus lié à la défense d'un certain point de vue, à une démonstration argumentative orientée par la théorie.

5 Les lecteurs et lectrices trouveront dans Morrissette (2009, 2010a) les détails concernant chacune de ces sous-catégories, ainsi que les extraits issus des entretiens s'y rapportant. 
${ }^{6}$ Plus précisément, la technique d’impact a consisté à envoyer deux élèves à la salle du personnel-enseignant dans le but d'aller y chercher un «bouli », un objet fictif pour lequel l'enseignante n’a donné aucune indication. D’après l’épisode narré, les élèves se seraient précipités à la salle du personnel-enseignant pour revenir quelques minutes plus tard, sans avoir trouvé l'objet en question. C'est seulement à ce moment-là que l'enseignante aurait établi un lien entre cette quête vaine et la tâche sur les polygones, insistant sur le fait qu'il est préférable de savoir ce qu'on cherche avant de se lancer dans la réalisation d'une tâche, et donc de signaler les incompréhensions.

\section{Références}

Beaulieu, D. (2006). Techniques d'impact pour grandir: illustrations pour développer l'intelligence émotionnelle chez les enfants. Québec: Le Gardeur.

Becker, H. S. (1985). Outsiders. Études de sociologie de la déviance (trad. J.P. Briand \& J.- M. Chapoulie). Paris : Éditions A.- M. Métailié. (Ouvrage original publié en 1963).

Becker, H. S. (1986). Doing things together. Evanston, IL : Northwestern University Press.

Becker, H. S. (2002). Les ficelles du métier : comment conduire sa recherche en sciences sociales. Paris : La Découverte.

Becker, H. S. (2006a). Le travail sociologique. Méthode et substance. Fribourg, $\mathrm{CH}$ : Academic Press Fribourg.

Becker, H. S. (2006b). Les mondes de l'art (trad. J. Bouniort) ( $2^{\mathrm{e}}$ éd.). Paris : Flammarion. (Ouvrage original publié en 1982.)

Becker, H. S., \& McCall, M. M. (Éds). (1990). Symbolic interaction and cultural studies. Chicago : University of Chicago Press.

Berger, P., \& Luckmann, T. (1986). La construction sociale de la réalité (trad. P. Taminiaux). Paris : Méridiens-Klincksieck.

Blanchet, A., \& Gotman, A. (1992). L'enquête et ses méthodes : l'entretien. Paris : Nathan Université.

Blumer, H. (1937). Social psychology. Dans E. P. Schmidt (Éd.), Man and society: a substantive introduction to the social science (pp. 144-198). New York : Prentice-Hall.

Blumer, H. (1969). Symbolic interactionism: perspective and method. Englewood Cliffs, NJ : Prentice Hall. 
Boden, D. (1990). People are talking: conversation analysis and symbolic interaction. Dans H. S. Becker, \& M. M. McCall (Éds), Symbolic interaction and cultural studies (pp. 244-274). Chicago, IL : University of Chicago Press.

Callon, M., Lascoumes, P., \& Barthe, P. (2001). Agir dans un monde incertain : essai sur la démocratie technique. Paris : Seuil.

Chapoulie, J.- M. (2001). La tradition sociologique de Chicago. 1892-1961. Paris : Seuil.

Chapoulie, J.- M. (2008). Malentendus transatlantiques. La tradition de Chicago, Park et la sociologie française. L'Homme, 187-188(3-4), 223-246.

Coulon, A. (1992). L'École de Chicago. Paris: Presses universitaires de France.

Darré, J.- P. (1999). La production de connaissance pour l'action. Arguments contre le racisme de l'intelligence. Paris: Éditions de la Maison des sciences de l'homme et Institut National de la Recherche Agronomique.

De Certeau, M. (1990). L'invention au quotidien (Vol. 1). Paris : Gallimard.

Demazière, D. (2008). L'entretien biographique comme interaction. Négociations, contre-interprétations, ajustement de sens. Langage et société, 123, 15-35.

Demazière, D., \& Dubar, C. (1997). Analyser les entretiens biographiques. L'exemple des récits d'insertion. Paris : Nathan.

De Queiroz, J- M., \& Ziolkowski, M. (1997). L'interactionnisme symbolique. Rennes, FR : Presses Universitaires de Rennes.

Desgagné, S. (1997). Le concept de recherche collaborative: l'idée d'un rapprochement entre chercheurs universitaires et praticiens enseignants. Revue des sciences de l'éducation, 23(2), 371-393.

Desgagné, S. (1998). La position du chercheur en recherche collaborative : illustration d'une démarche de médiation entre culture universitaire et culture scolaire. Dans C. Deschamps, \& R. Letendre (Éds), Recherches qualitatives, 18: L'attitude du chercheur en recherche qualitative (pp. 77-105). Québec : Université du Québec à Trois-Rivières.

Desgagné, S. (2001). La recherche collaborative: nouvelle dynamique de recherche en éducation. Dans M. Anadón (Éd.), Nouvelles dynamiques de recherche en éducation (pp. 51-76). Québec: Presses de l'Université Laval. 
Duchesne, S., \& Haegel, F. (2005). L'entretien collectif (2 ${ }^{\mathrm{e}}$ éd.). Paris : Armand Colin.

Fourez, G. (2003). Apprivoiser l'épistémologie. Bruxelles : De Boeck.

Giddens, A. (1987). La constitution de la société (trad. M. Audet). Paris : Presses universitaires de France.

Glaser, B., \& Strauss, A. (2010). La découverte de la théorie ancrée (trad. P. Paillé). Paris : Armand Colin. (Ouvrage original publié en 1967).

Goffman, E. (1968). Asiles : études sur la condition sociale des malades mentaux et autres reclus. Paris : Minuit.

Goffman, E. (1973). La mise en scène de la vie quotidienne (Vol. 2). Paris : Minuit.

Goffman, E. (1987). Façons de parler (trad. A. Kihm). Paris : Minuit.

Gumperz, J. (1989). Engager la conversation. Introduction à la sociolinguistique interactionnelle. Paris : Minuit.

Hughes, E. C. (1951). Mistakes at work. The Canadian Journal of Economics and Political Science / Revue canadienne d'économie et de science politique, 17(3), 320-327.

Hughes, E. C. (1996). Le regard sociologique. Essais choisis (trad. J.- M. Chapoulie). Paris : École des Hautes Études en Sciences Sociales.

Hunter, F. (1953). Community power structure. A study of decision makers. Chapel Hill, NC : University of North California Press.

Kerbrat-Orecchioni, C. (1996). La conversation. Paris : Seuil.

Kerbrat-Orecchioni, C. (2005). Le discours en interaction. Paris: Armand Colin.

Legendre, M.- F. (2001). Approches constructivistes et nouvelles orientations curriculaires. D'un curriculum fondé sur l'approche par objectifs à un curriculum axé sur le développement de compétences. Dans P. Jonnaert, \& D. Masciotra (Éds), Constructivisme et choix contemporains. Hommage à Ernst von Glasersfeld (pp. 51-91). Montréal : Presses de l’Université du Québec.

Lessard, C., \& Tardif, M. (1999). Le travail enseignant au quotidien : expérience, interactions humaines et dilemmes professionnels. Québec: Presses de l’Université Laval.

Lieberman, A. (1986). Collaborative research: working with, not working on... Educational Leadership, 43(5), 28-32. 
Mishler, E. G. (1986). Research interviewing. Cambridge, MA : Harvard University Press.

Morrissette, J. (2002). Évolution de la conception de l'évaluation formative des apprentissages à travers le discours ministériel depuis 1981 (Mémoire de maîtrise inédit). Université du Québec en Abitibi-Témiscamingue en association avec l'Université du Québec à Rimouski, AbitibiTémiscamingue.

Morrissette, J. (2009). Manières de faire l'évaluation formative des apprentissages selon un groupe d'enseignantes du primaire: une perspective interactionniste (Thèse de doctorat inédite). Université Laval, Québec.

Morrissette, J. (2010a). Manières de faire l'évaluation formative des apprentissages : analyse interactionniste du savoir-faire d'enseignantes du primaire. Sarrebruck, CH : Les éditions universitaires européennes.

Morrissette, J. (2010b). Une perspective interactionniste : un autre point de vue sur l'évaluation des apprentissages. Sociologies. Repéré à http://sociologies.revues.org/index3028.html

Morrissette, J. (2011). Ouvrir la boîte noire de l'entretien de groupe. Recherches qualitatives, 29(3), 7-32.

Morrissette, J., \& Desgagné, S. (2009). Le jeu des positions de savoir en recherche collaborative: une analyse des points de vue négociés d'un groupe d'enseignantes du primaire. Recherches qualitatives, 28(2), 118144.

Pépin, Y. (1994). Savoirs pratiques et savoirs scolaires : une représentation constructiviste de l'éducation. Revue des sciences de l'éducation, 20(1), 63-85.

Pessin, A. (2004). Un sociologue en liberté. Lecture de Howard S. Becker. Québec : Presses de l’Université Laval.

Poupart, J. (1997). L'entretien de type qualitatif: considérations épistémologiques, théoriques et méthodologiques. Dans J. Poupart, L.- H. Groulx, A. Laperrière, R. Mayer, \& A. P. Pires (Éds), La recherche qualitative. Enjeux épistémologiques et méthodologiques (pp. 173-209). Montréal : Gaëtan Morin.

Schön, D. A. (1983). The reflective practitioner. New York : Basic Books. 
Schön, D. A. (1996). À la recherche d'une nouvelle épistémologie de la pratique et de ce qu'elle implique pour l'éducation des adultes. Dans J.- M. Barbier (Éd.), Savoirs théoriques et savoirs d'action (pp. 201-222). Paris : Presses universitaires de France.

Strauss, A. L. (1971). Professions, work and careers. San Francisco: Sociology Press.

Thomas, W. I. (1923). The unadjusted girl. Boston : Brown \& Co.

Thomas, W. I., \& Znaniecki, F. (1918-1920). The polish peasant in Europe and America, (Vol. 1-5). Boston : The Gorham Press.

Tochon, F. V. (1996). Rappel stimulé, objectivation clinique, réflexion partagée. Fondements méthodologiques et applications pratiques de la rétroaction vidéo en recherche et en formation. Revue des sciences de l'éducation, 22(3), 467-502.

Touraine, A. (1984). Le retour de l'acteur. Paris : Fayard.

Traverso, V. (1999) L'analyse des conversations. Paris : Nathan.

Van Campenhoudt, L., Chaumont, J.- M., \& Franssen, A. (2005). La méthode d'analyse en groupe. Application aux phénomènes sociaux. Paris : Dunod.

Van der Maren, J.- M. (2004). Méthodes de recherche pour l'éducation ( $2^{\mathrm{e}}$ éd.). Montréal : Presses de l’Université de Montréal.

Wenger, E. (2005). La théorie des communautés de pratique. Apprentissage, sens et identité (trad. F. Gervais). Québec : Presses de l'Université Laval.

Wittgenstein, L. (2004). Recherches philosophiques (trad. F. Dastur, M. Élie, J.- L. Gautero, D. Janicaud \& É. Rigal). Paris : Gallimard.

Wittgenstein, L. (2006). De la certitude (trad. D. Moyal-Sharrock). Paris : Gallimard.

Woods, P. (1992). Symbolic interactionnism : theory and method. Dans M. D. Le Compte, W. L. Millroy, \& J. Preissle (Éds), The handbook of qualitative research (pp. 337-404). New York: Academic Press.

Joëlle Morrissette est professeure adjointe à la Faculté des sciences de l'éducation de l'Université de Montréal. Ses travaux s'inscrivent dans les domaines de l'évaluation des apprentissages, des perspectives interactionnistes ainsi que des savoirs pratiques, et portent la marque d'une préoccupation particulière pour l'épistémologie de la recherche. Sur le plan méthodologique, elle privilégie les investigations collaboratives sur le terrain par le biais d'entretiens individuels et de groupe avec les enseignantes et les enseignants. Sur le plan analytique, elle aborde ses objets d'étude sous l'angle des interactions qui lient les acteurs de la situation éducative au quotidien, cherchant à 
32 Recherches Qualitatives / Vol. 30(1)

rendre compte des significations qu'ils engagent dans ces interactions. À cette fin, et concevant que le sens est indissociable de la manière dont il est produit, elle recourt à des analyses de discours, plus précisément à l'analyse de conversations. 